\title{
Internationale Entwicklungen und Trends in der medizinischen Ästhetik
}

\begin{abstract}
Der Trend hin zu minmal-invasiven Methoden der optischen Hautverjüngung, wie die Faltenbehandlung mit Hyaluron oder Botulinum, ist weiterhin ungebrochen. Dabei steigt nicht nur die Nachfrage von Seiten der Patienten stetig, sondern auch Indikationen, Behandlungsmöglichkeiten und Verfahren werden international fortlaufend weiterentwickelt.
\end{abstract}

Während die Sichtweise der optischen Hautverjüngung zu Beginn auf die Reduktion einzelner Falten beschränkt war, betrachtet man mittlerweile die gesamte äußere Entscheidung des Gesichts, insbesondere die Volumenverteilung, das individuelle Hautbild und die an das Gesicht angrenzenden Regionen wie Hals und Dekolleté. Auch eine auf das Alter und jeweilige Stadium der Hautalterung abgestimmte Therapie ist für eine fachlich kompetente Behandlung notwendig und wird von dem zunehmend aufgeklärteren Patienten aktiv eingefordert. Für den ästhetisch arbeitenden Arzt wird es zeitaufwändiger, sich über neue Trends und Entwicklungen zu informieren und diese fachlich kompetent in den Praxisalltag zu integrieren.

\section{Q-Med/ Galderma Ästhetiktag}

Das Unternehmen Q-Med/ Galderma hat diese Entwicklungen frühzeitig antizipiert und veranstaltet bereits zum zweiten Mal in Folge einen Kompetenztag der ästhetischen Dermatologie. Unter dem Motto „Science \& Practice for Excellence" werden im Rahmen der 23. Münchener Fortbildungswoche für praktische Dermatologie und Venerologie in München international anerkann- te Experten aus Wissenschaft und Praxis einen ganzen Tag lang dezidierte Einblicke geben in die neuesten Erkenntnisse und Behandlungsoptionen mit den kompletten Fillersortimenten Restylane ${ }^{\circ}$ und Emervel $^{\circ}$, sowie dem Botulinum Azzalure ${ }^{\circ}$ Im Fokus stehen dabei das Anpassen der Therapie auf verschiedene Altersstufen, die Kombination unterschiedlicher Produkte bei einer Full Face Behandlung, neueste Injektionstechniken sowie das Erstellen von optimierten Behandlungsprotokollen und entsprechender Kasuistik. „Für interessierte Fachärzte stellt dies eine einzigartige Möglichkeit dar, sich innerhalb eines Tages und auf einer Veranstaltung umfassend und auf höchstem Niveau im Bereich der minimal-invasiven Ästhetik fortzubilden," empfiehlt Frau Dr. Tatjana Pavicic aus München, die zusammen mit Herrn Dr. Gerhard Sattler, Darmstadt, bereits zum zweiten Mal der diesjährigen Firmenveranstaltung am 22. Juli in München vorsitzt.

Anmeldung zur Veranstaltung direkt unter www. fortbildungswoche.de

Nach Informationen der Galderma Laboratorium $\mathrm{GmbH}$

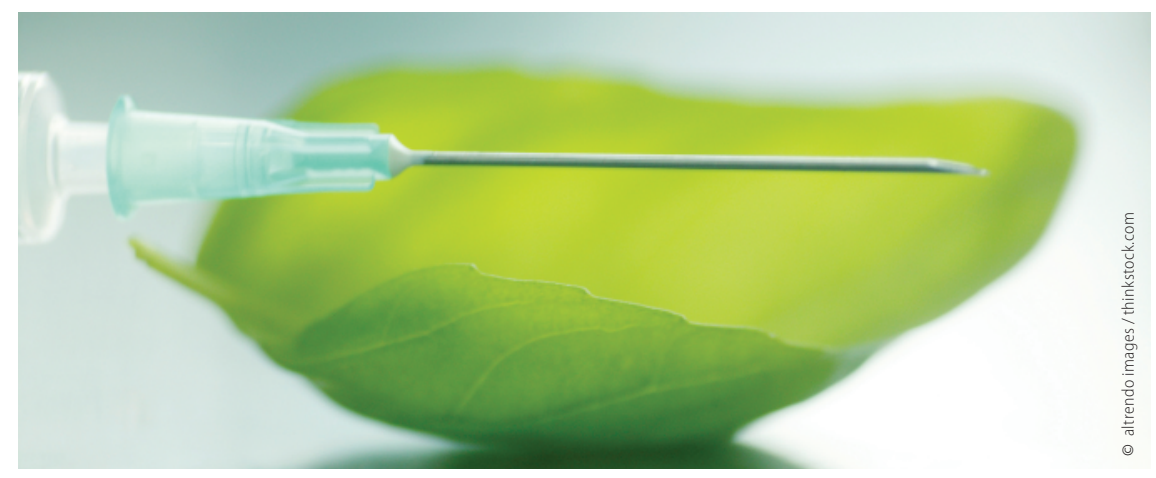

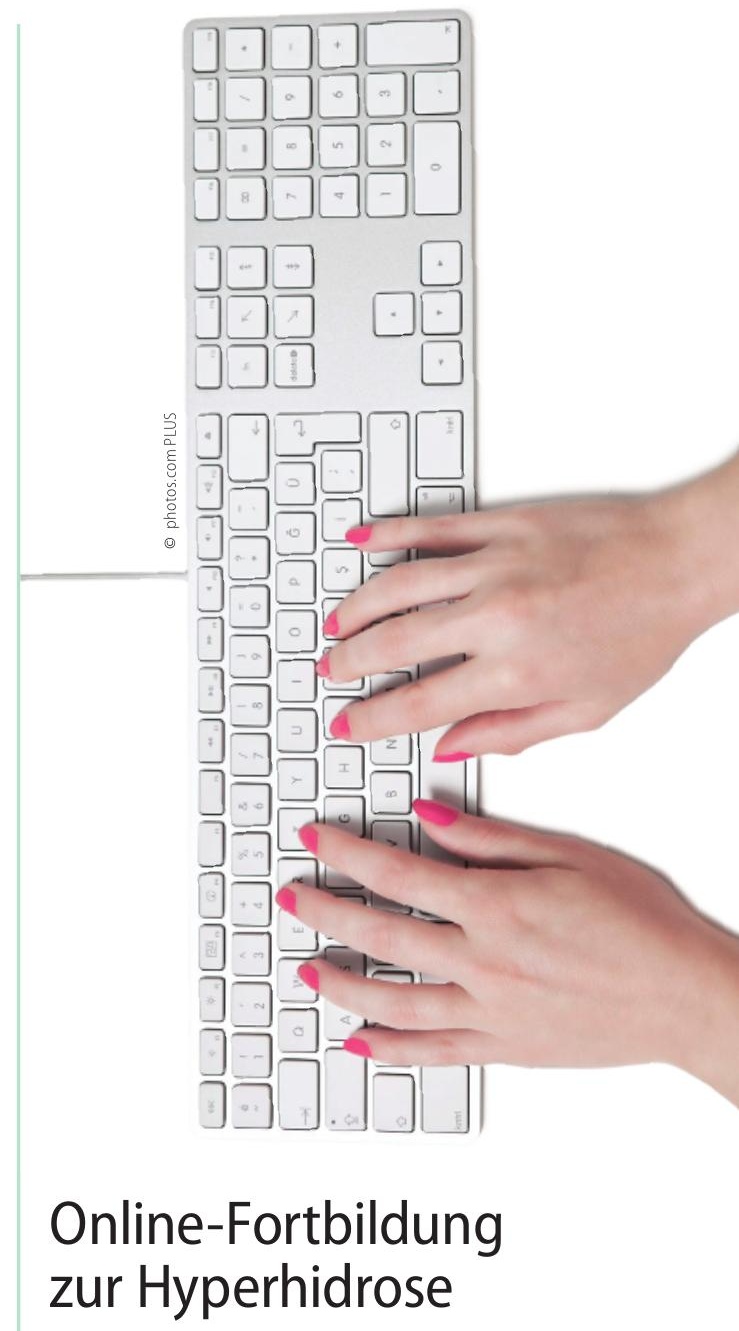

Das Unternehmen Riemser hat im April unter der Adresse www.fortbildung-riemser.de das interaktive Lernmodul „Hyperhidrose - das krankhafte Schwitzen“ins Netz gestellt. Die Online-Fortbildung informiert über die Epidemiologie und Pathogenese der Hyperhidrose sowie über die Therapie mit Vagantin ${ }^{\circledR}$

Viele Patienten zögern, sich mit übermäßigem Schwitzen an ihren Arzt zu wenden - aus Scham oder weil sie die Krankheit nicht kennen. Dabei reduziert eine Therapie der Hyperhidrose signifikant die Schwitzneigung und verbessert erheblich die Lebensqualität. Wenn Sie diese Patienten erkennen und beraten wollen, dann nehmen Sie sich die zehn Minuten Zeit für die Online-Fortbildung. Zur Teilnahme benötigen Sie entweder einen Zugangscode, den Sie beim Riemser-Außendienst erhalten können, oder einfach ein Springer Medizin Login.

(Red) 